\title{
Síndrome de burnout em profissionais de enfermagem
}

\author{
Burnout syndrome in nursing professionals
}

\author{
Síndrome de Burnout en los profesionales de enfermería
}

Márcia Karênina Passos de Sousa1 ${ }^{1}$, Valdenis da Silva Lima1 ${ }^{1}$, Maria Tamires Alves Ferreira², Tatiana Naiana Rodrigues dos Santos Porto ${ }^{1}$, Luciana Stanford Baldoino ${ }^{2 *}$, Vinícius de Sousa Martins², Dorivaldo Pereira Carvalho², Sônia Maria Leite Alcântara².

\section{RESUMO}

Objetivo: Revisar os fatores relacionados à manifestação da Síndrome de Burnout em trabalhadores de enfermagem. Métodos: Trata-se de uma revisão integrativa da literatura. A coleta foi realizada de fevereiro a setembro de 2017. Tendo como critérios de inclusão artigos em português, inglês e espanhol apresentados na íntegra online e publicados de 2013 a 2017. Resultados: De acordo com os artigos elencados no estudo verificou-se que, a Síndrome de Burnout (SB) acomete principalmente profissionais de enfermagem do sexo feminino, indivíduos em idade jovem e casados, sendo que têm grande chance de predisposição ao desenvolvimento desta síndrome, por serem os profissionais de saúde que mais tempo ficam em contato com o paciente e seus familiares. Além disso, no âmbito hospitalar, esses profissionais são submetidos a condições insalubres e precárias de trabalho, que geram uma baixa qualidade de vida, como também geram consequências à instituição empregadora, na medida em que os efeitos se refletem na produtividade deste profissional, na eficiência e na organização, nos custos com no tratamento de saúde dos funcionários, na contratação e no treinamento de novos trabalhadores. Considerações finais: Evidenciou-se, a necessidade de diagnósticos mais precisos e precoces, além do estabelecimento de medidas preventivas e de tratamento para a SB.

Palavras-chave: Esgotamento Profissional, Doenças Profissionais, Enfermagem.

\begin{abstract}
Objective: Review the factors related to the manifestation of Burnout Syndrome in nursing workers. Methods: This is an integrative literature review. The collection was carried out from February to September 2017. The inclusion criteria were articles in Portuguese, English and Spanish presented in full online and published from 2013 to 2017. Results: According to the articles listed in the study, it was found that the Burnout Syndrome (BS) mainly affects female nursing professionals, young people and married, and they have a high chance of predisposition to the development of this syndrome, because health professionals are in longer contact with the patient and your family members. Moreover, in the hospital environment, these professionals are subjected to unhealthy and precarious working conditions, which generate a low quality of life, as well as consequences for the employing institution, as the effects are reflected in the productivity of this professional, the efficiency and in the organization, costs of employee health care, hiring and training of new workers. Final considerations: It was evidenced the need for more accurate and early diagnoses, besides the establishment of preventive and treatment measures for BS.
\end{abstract}

Key words: Burnout, Occupational Diseases, Nursing.

${ }^{1}$ Enfermeira, Graduação em Enfermagem pela Faculdade de Ensino Superior Múltiplo - IESM, Timon-MA.

2 Docentes da Faculdade de Ensino Superior Múltiplo - IESM, Timon-MA. *E-mail: Isbaldoino@hotmail.com

SUBMETIDO EM: $8 / 2019$

ACEITO EM: 9/2019

PUBLICADO EM: 10/2019

REAS/EJCH | Vol.Sup.34 | e1413 | DOI: https://doi.org/10.25248/reas.e1413.2019 Página 1 de 9 


\section{RESUMEN}

Objetivo: Resiva los factores relacionados con la manifestación del síndrome de Burnout en trabajadores de enfermería. Métodos: Esta es una revisión de literatura integradora. La colección se realizó de febrero a septiembre de 2017. Los criterios de inclusión fueron artículos en portugués, inglés y español presentados en línea en su totalidad y publicados de 2013 a 2017. Resultados: De acuerdo con los artículos enumerados en el estudio, se encontró que El Síndrome de Burnout (BS) afecta principalmente a profesionales de enfermería, jóvenes y casados, y tienen una alta probabilidad de predisposición al desarrollo de este síndrome, porque los profesionales de la salud tienen un contacto más prolongado con el paciente y los miembros de tu familia Además, en el entorno hospitalario, estos profesionales están sujetos a condiciones de trabajo poco saludables y precarias, que generan una baja calidad de vida, así como consecuencias para la institución empleadora, ya que los efectos se reflejan en la productividad de este profesional, la eficiencia y en la organización, los costos de la atención médica de los empleados, la contratación y la capacitación de nuevos trabajadores. Consideraciones finales: Se evidenció la necesidad de diagnósticos más precisos y tempranos, además del establecimiento de medidas preventivas y de tratamiento para BS.

Palabras clave: Agotamiento, Enfermedades Profesionales, Enfermería.

\section{INTRODUÇÃO}

A síndrome de Burnout (SB) é caracterizada como um fenômeno psicossocial que ocorre como resposta crônica aos estressores interpessoais nas situações de trabalho, atingindo principalmente aqueles que trabalham em locais agitados, desgastantes e sob pressão, onde muitas vezes o profissional não valorizado. As suas consequências surgem deste ritmo atual, levando a um estado de tensão emocional e crônico (SANTOS AVS, et al., 2016).

A SB se manifesta de forma crônica, por meio de desequilíbrios emocionais devido a maiores exigências no trabalho, sendo estas desproporcionais ao que indivíduo pode suportar. Alguns autores conceituam esta patologia como uma formulação tridimensional: exaustão emocional, que tem como característica a ausência ou deficiência de energia, animação e um sentimento de esgotamento (LIMA AS, 2016).

Essa patologia é relacionada com o sentimento de fracasso e exaustão, causando danos físicos e psicológicos muitas vezes incapacitantes. Os principais sintomas são: agressividade, isolamento, mudança de humor, irritabilidade, dificuldade de concentração, falta de memória, ansiedade, baixa auto-estima, tristeza, pessimismo, absenteísmo, entre outros. Atinge geralmente os profissionais que têm ou mantêm contato direto com pessoas, principalmente os profissionais da saúde (FRANÇA TLB, et al., 2014).

O Ministério da Previdência Social do Brasil contabilizou que cerca de 4,2 milhões de pessoas foram afastadas do trabalho. Destas 3.852 receberam diagnóstico da síndrome. Segundo as leis de auxílio do trabalho dos brasileiros a síndrome de Burnout se classifica de acordo com a classificação de doenças (CID 10, como Z73-0), porque torna o indivíduo incapaz de trabalhar e gera inúmeros custos e prejuízos a quem é acometido e à sociedade (CARRARA GLR, et al., 2015).

Os serviços de atenção à saúde contêm fatores de risco elevados que potencializam situações de Burnout, pois estão acostumados a seguir a lógica de produção, mesmo com recursos escassos, o que dificulta 0 trabalho do profissional, aumentando assim o estresse e sobrecarga do trabalhador. Dessa forma, essas dificuldades interferem na qualidade de vida do trabalhador, principalmente quando se trata de setor público, onde se vê refletido o cotidiano brasileiro (RODRIGUES UM e RIBEIRO ER, 2014).

Destaca-se, o interesse surgiu em realizar esta pesquisa sobre a síndrome de Burnout (SB) devido esta patologia ser considerada um problema de saúde pública, que vem se mostrado cada vez mais frequente, atingindo principalmente profissionais da área da saúde, em especial os enfermeiros por estarem presentes na linha de frente dos atendimentos, tendo que lidar com usuários cada vez mais exigentes, longas jornadas de trabalho, problemas nas relações sociais, condições de trabalho inadequadas, desvalorização profissional, entre outros. 
Dessa forma, os profissionais de saúde não estão imunes a esta doença pelo fato de terem conhecimentos neste campo, da mesma forma que o conhecimento não serve de substituto à necessidade do apoio emocional quando se trata do sofrimento e da doença. Os locais de trabalho também acabam sendo prejudicados pela ocorrência desta doença, visto que o grande número de faltas e de profissionais afetados tendem a diminuir a eficiência e qualidade do serviço oferecido (CAMPOS ICS, et al., 2015).

Assim, o estudo ajudará a compreender melhor esta patologia e a fornecer elementos que sirvam de subsídio para o enfrentamento de problemas da profissão relacionados à síndrome de Burnout, tais como insatisfação profissional, exaustão emocional, despersonalização, absenteísmo e doenças relacionadas como a depressão, o alcoolismo e o tabagismo. Doenças que podem ser relacionadas ao trabalho, mas muitas vezes são vistas como subterfúgios para descanso e férias. Diante do exposto, o objetivo deste estudo foi revisar os fatores relacionados à manifestação da SB em trabalhadores de enfermagem.

\section{MÉTODOS}

O presente trabalho trata-se de uma revisão integrativa da literatura, método de pesquisa que permite a busca, a avaliação e a síntese de várias evidências encontradas com o tema investigado, onde o produto final do estudo será o conhecimento do tema proposto, e que dá possibilidade ao leitor de ter o acesso a diversas pesquisas em um só estudo (MENDES KDS et al., 2008).

No entanto, a revisão integrativa de literatura baseia-se em etapas que se unem para o objetivo final, são elas: a identificação de tema e onde se seleciona a questão norteadora da pesquisa, o estabelecimento dos critérios de inclusão e de exclusão, a identificação dos estudos pré- selecionados, categorização dos estudos selecionados, análise e interpretação dos resultados e apresentação da síntese do conhecimento (MENDES KDS, et al., 2008).

Para a realização do levantamento bibliográfico, fez-se uma busca utilizando os descritores em saúde "esgotamento profissional", "enfermagem", "doenças profissionais" e "transtornos mentais". Tendo como critérios de inclusão foram artigos em português, inglês e espanhol apresentados na íntegra online e publicados no período de 2013 a 2017. Foram excluídos livros, cartas, resenhas, artigos reflexivos e trabalhos de revisão de literatura, artigos que não condiziam com o tema proposto, bem como indisponíveis para download e artigos incompletos. Para restringir a busca de dados neste estudo, foram utilizados os Operadores Booleanos (Tabela 1).

Tabela 1 - Estratégia de Busca utilizando Operadores Booleanos.

\begin{tabular}{|c|l|c|c|c|}
\hline Esgotamento profissional & AND & Enfermagem & \\
\hline Doenças profissionais & OR & Esgotamento profissional & AND & Transtornosmentais \\
\hline Doenças profissionais & AND & \multicolumn{2}{|c|}{ Enfermagem } \\
\hline
\end{tabular}

Fonte: Sousa MKP, Porto TNRS, Lima VS, et al., 2017.

O levantamento bibliográfico foi realizado de fevereiro a setembro de 2017 nas bases de dados Sistema Latino-Americano e do Caribe de Informação em Ciências da Saúde (Lilacs), Scientific Eletronic Library Online (Scielo), Medical Literature Analysis and Retrivel System Online (Medline) e Pubmed. Foram encontrados nove artigos finais, sendo: base de dados Scielo (n: 8), Lilacs (n: 8), Medline (n: 3), Pubmed (n: 1), que foram utilizados na elaboração dos resultados.

Para chegar a este desfecho foram aplicados os critérios de exclusão, sendo eles: na primeira exclusão, artigos duplicados e título incompatível com o tema Síndrome de Burnout em enfermeiros, na segunda exclusão, artigos que não abordavam a Síndrome de Burnout em Profissionais da Saúde e na terceira exclusão, artigos com metodologia de revisão de literatura e de reflexão. Com o objetivo de esclarecer estas etapas, foi elaborado um fluxograma (Figura 1). 
Figura 1- Fluxograma da aplicação das etapas dos critérios de inclusão e exclusão dos artigos para amostra final.

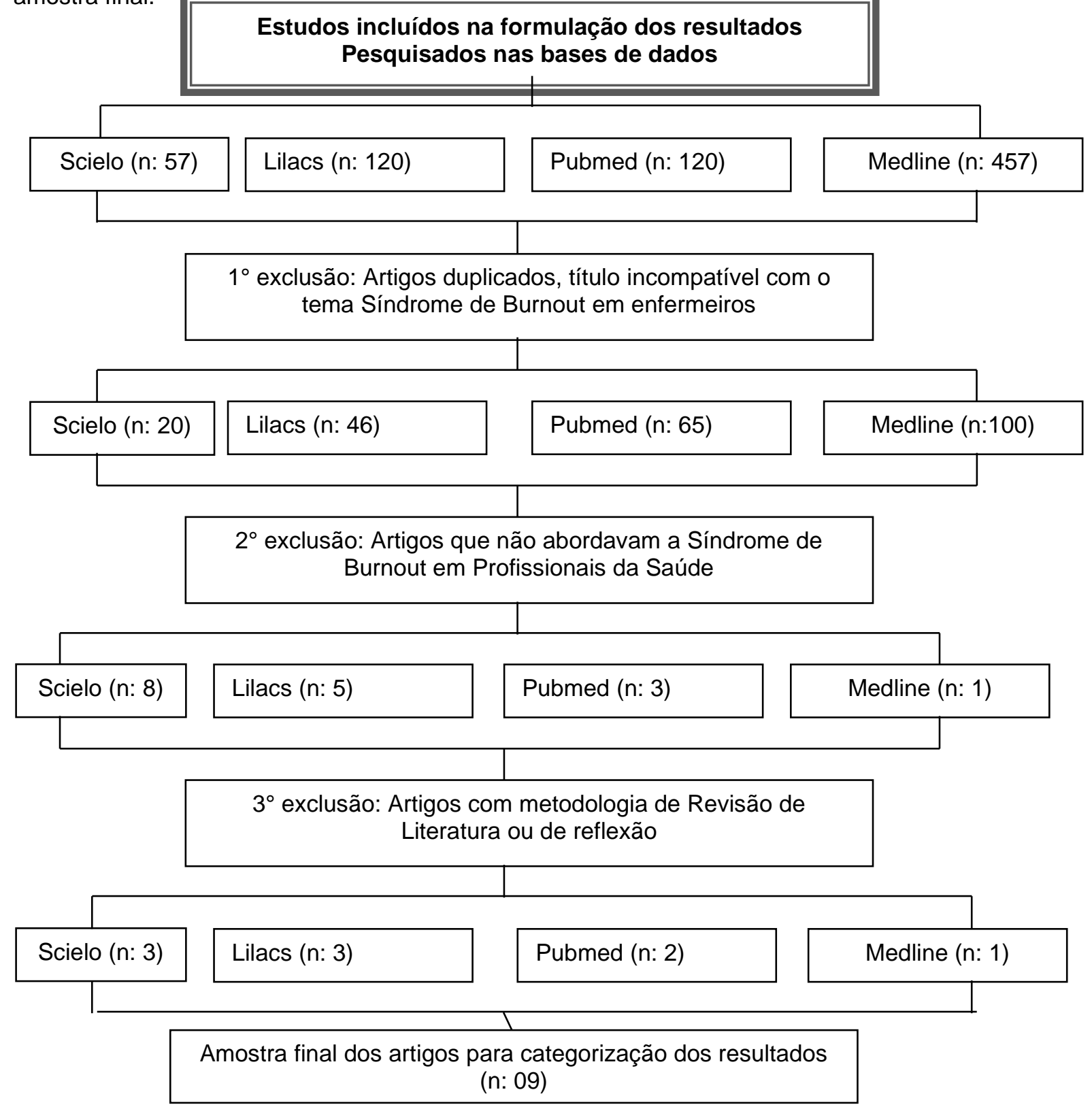

Fonte: Sousa MKP, Porto TNRS, Lima VS, et al., 2017.

\section{RESULTADOS E DISCUSSÃO}

A análise dos resultados foi realizada de maneira descritiva em formato de tabela e texto, onde foram catalogados os fatores influentes na ocorrência e manifestação da Síndrome de Burnout em enfermeiros. Para a análise dos artigos utilizou-se as variáveis: Tipo de estudo, Autor/ano, Base de dados, Periódico publicado, Qualis Capes e Síntese dos resultados. Vale salientar que parte dos dados foi apresentada em formato de quadro esquemático, mostrando a síntese das principais características dos estudos analisados, e em seguida foi apresentada de forma descritiva para um melhor entendimento do conteúdo apresentado nos artigos. Em seguida, foi realizada análise e discussão dos resultados fundamentada no pensamento dos autores sobre a temática. É pertinente enfocar que a maioria dos artigos foram publicados em revistas especializadas e que a avaliação das evidências encontradas ocorreu mediante leitura cuidadosa dos artigos e do fichamento individual dos mesmos por meio de um instrumento construído anteriormente. Foram selecionados nove artigos no estudo e que somente após analisados na íntegra foi elaborado o Quadro 1. 
Quadro 1: Artigos incluídos no estudo baseado no:Título do artigo, Tipo de estudo, Autor, Base de dados, Períodico publicado/ ano, Qualis Capes e Síntese dos resultados. Timon-MA, 2017.

\begin{tabular}{|c|c|c|c|c|c|c|c|}
\hline $\mathbf{N}^{\circ}$ & Título do artigo & $\begin{array}{l}\text { Tipo de } \\
\text { estudo }\end{array}$ & Autor & $\begin{array}{l}\text { Base de } \\
\text { dados }\end{array}$ & $\begin{array}{c}\text { Períodico de } \\
\text { publicação/ ano }\end{array}$ & $\begin{array}{l}\text { Qualis } \\
\text { Capes }\end{array}$ & Síntese dos resultados \\
\hline E1 & $\begin{array}{l}\text { Burnout: o impacto da } \\
\text { satisfação no trabalho } \\
\text { em profissionais de } \\
\text { enfermagem }\end{array}$ & $\begin{array}{l}\text { Abordagem } \\
\text { quantitativa }\end{array}$ & $\begin{array}{l}\text { Sá MAS, } \\
\text { Silva PO, } \\
\text { Funchal B. }\end{array}$ & Lilacs & $\begin{array}{c}\text { Psicologia \& Sociedade / } \\
2014\end{array}$ & B3 & $\begin{array}{l}\text { O fator satisfação com o ambiente reduz a } \\
\text { exaustão emocional; o aumento com a } \\
\text { satisfação com o trabalho e oportunidade de } \\
\text { crescimento reduz as dimensões exaustão } \\
\text { emocional e despersonalização. }\end{array}$ \\
\hline E2 & $\begin{array}{l}\text { Ocorrência da } \\
\text { síndrome de Burnout } \\
\text { em enfermeiros } \\
\text { residentes }\end{array}$ & $\begin{array}{c}\text { Estudo } \\
\text { Transversal }\end{array}$ & $\begin{array}{l}\text { Tavares } \\
\text { KFA, et al. }\end{array}$ & Scielo & Acta Paul Enferm / 2014 & B2 & $\begin{array}{l}\text { A ocorrência da síndrome de Burnout foi } \\
\text { identificada no grupo de residentes de } \\
\text { enfermagem, os } \\
\text { quais apresentaram os seguintes fatores } \\
\text { determinantes: indivíduos jovens, do gênero } \\
\text { feminino, solteiros, sem filhos, recém-formados } \\
\text { e inseridos em setores de alta complexidade. }\end{array}$ \\
\hline E3 & $\begin{array}{l}\text { Sentimentos de } \\
\text { Enfermeiros Frente ao } \\
\text { Paciente Oncológico }\end{array}$ & $\begin{array}{l}\text { Exploratório } \\
\text { com } \\
\text { abordagem } \\
\text { qualitativa }\end{array}$ & $\begin{array}{l}\text { Kolhs M, et } \\
\text { al. }\end{array}$ & Pubmed & J Health Sci / 2016 & B1 & $\begin{array}{l}\text { Neste estudo, a maioria dos enfermeiros } \\
\text { entrevistados relataram sentir um grande } \\
\text { desgaste emocional, sentimentos ruins, } \\
\text { sentimentos e estratégias defensivas, } \\
\text { características tipícas da Síndrome de Burnout. }\end{array}$ \\
\hline E4 & $\begin{array}{l}\text { Risco de adoecimento } \\
\text { dos profissionais de } \\
\text { enfermagem } \\
\text { noTrabalho em } \\
\text { atendimento móvel de } \\
\text { urgência }\end{array}$ & $\begin{array}{c}\text { Estudo } \\
\text { Transversal }\end{array}$ & $\begin{array}{l}\text { Worm FA, } \\
\text { et al. }\end{array}$ & Scielo & Rev Cuid / 2016 & B2 & $\begin{array}{l}\text { Na avaliação do contexto de trabalho, a } \\
\text { organização do trabalho foi apontada como } \\
\text { crítica. Os Indicadores Prazer e Sofrimento no } \\
\text { Trabalho apontaram liberdade de expressão } \\
\text { dos profissionais, por outro lado há falta de } \\
\text { reconhecimento e esgotamento profissional. } \\
\text { Para a avaliação dos danos relacionados ao } \\
\text { trabalho destacam-se os danos físicos. }\end{array}$ \\
\hline E5 & $\begin{array}{l}\text { A Síndrome de burnout } \\
\text { em trabalhadores de } \\
\text { enfermagem em dois } \\
\text { hospitais no sul do } \\
\text { Chile }\end{array}$ & $\begin{array}{l}\text { Estudo descriti- } \\
\text { vo, transversal } \\
\text { e comparativo }\end{array}$ & $\begin{array}{l}\text { Palma FS, } \\
\text { Suazo SV. }\end{array}$ & Medline & Av Enferm / 2016 & B5 & $\begin{array}{l}\text { A amostra apresentou uma classificação baixa } \\
\text { e média nas subescalas Desgaste emocional e } \\
\text { Cinismo, e uma alta classificação na subescala } \\
\text { Eficácia profissional. }\end{array}$ \\
\hline
\end{tabular}

REAS/EJCH | Vol.Sup.34 | e1413 | DOI: https://doi.org/10.25248/reas.e1413.2019 Página 5 de 9 


\begin{tabular}{|c|c|c|c|c|c|c|c|}
\hline E6 & $\begin{array}{l}\text { Stress, burnout e } \\
\text { avaliação cognitiva: } \\
\text { estudo na classe de } \\
\text { enfermagem }\end{array}$ & $\begin{array}{c}\text { Estudo } \\
\text { exploratório, } \\
\text { Transversal }\end{array}$ & $\begin{array}{l}\text { Pereira } \\
\text { MMA, } \\
\text { Gomes } \\
\text { ARS. }\end{array}$ & Lilacs & $\begin{array}{l}\text { Arquivos Brasileiros de } \\
\text { Psicologia / } 2015\end{array}$ & B5 & $\begin{array}{c}\text { Começando pela exaustão emocional, esta foi } \\
\text { predita pelo maior stress associado às relações } \\
\text { profissionais ao excesso de trabalho e à } \\
\text { carreira e remuneração, embora nesse caso as } \\
\text { diferenças tenham sido marginalmente } \\
\text { significativas. }\end{array}$ \\
\hline E7 & $\begin{array}{c}\text { Fatores } \\
\text { Sociodemográficos e } \\
\text { Ocupacionais } \\
\text { Associados à Síndrome } \\
\text { de Burnout em } \\
\text { Profissionais de } \\
\text { Enfermagem }\end{array}$ & $\begin{array}{c}\text { Estudo } \\
\text { comparativo, } \\
\text { transversal }\end{array}$ & $\begin{array}{l}\text { Campos, } \\
\text { ICM, } \\
\text { Angélico } \\
\text { AP, Oliveira } \\
\text { MS, et al. }\end{array}$ & Scielo & $\begin{array}{l}\text { Psychology / Psicologia: } \\
\text { Reflexão e Crítica / } 2015\end{array}$ & A2 & $\begin{array}{c}\text { Grande parte dos profissionais considerou que } \\
\text { o trabalho poderia gerar desgaste físico e } \\
\text { mental }(87,9 \%) \text {. Um terço dos trabalhadores } \\
(33,6 \%) \text { pensava em mudar } \\
\text { de profissão e a maioria apontou que, se } \\
\text { tivesse oportunidade, mudaria de emprego } \\
(68,1 \%) .\end{array}$ \\
\hline E8 & $\begin{array}{l}\text { Fatores psicossociais e } \\
\text { prevalência da } \\
\text { síndrome de burnout } \\
\text { entre trabalhadores de } \\
\text { enfermagem } \\
\text { intensivistas } \\
\end{array}$ & $\begin{array}{c}\text { Estudo } \\
\text { descritivo } \\
\text { seccional }\end{array}$ & $\begin{array}{l}\text { Silva JJL, et } \\
\text { al. }\end{array}$ & Pubmed & $\begin{array}{l}\text { Rev Bras Ter Intensiva / } \\
2015\end{array}$ & B3 & $\begin{array}{l}\text { A prevalência de síndrome de burnout foi de } \\
55,3 \%(n=72) \text {. Quanto aos quadrantes do } \\
\text { modelo demanda-controle, a baixa exigência } \\
\text { apresentou } 64,5 \% \text { de casos prevalentes } \\
\text { suspeitos e a alta exigência, } 72,5 \% \text { de casos ( } p \\
=0,006) \text {. }\end{array}$ \\
\hline E9 & $\begin{array}{l}\text { A síndrome de burnout } \\
\text { em profissionais da } \\
\text { Rede de Atenção } \\
\text { Primária à Saúde de } \\
\text { Aracaju, Brasil }\end{array}$ & $\begin{array}{c}\text { Estudo } \\
\text { transversal }\end{array}$ & $\begin{array}{l}\text { Silva SCPS, } \\
\text { et al. }\end{array}$ & Lilacs & $\begin{array}{l}\text { Ciência \& Saúde } \\
\text { Coletiva / } 2015\end{array}$ & B2 & $\begin{array}{c}\text { A prevalência da Síndrome de Burnout } \\
\text { pesquisada nos enfermeiros da Atenção } \\
\text { Básica, } \\
\text { foi de } 6,7 \% \text { a } 10,8 \% \text {, os fatores associados } \\
\text { foram } \\
\text { idade mais jovem, carga horária de trabalho } \\
\text { excessiva } \\
\text { e insatisfação profissional. Não houve diferença } \\
\text { entre as categorias avaliadas e a maioria } \\
\text { não apresenta a SB. No entanto, } 54,1 \% \\
\text { apresentaram } \\
\text { um risco elevado e moderado para desenvolver } \\
\text { essa síndrome, refletindo um processo de } \\
\text { adoecimento } \\
\text { que ameaça o bem-estar dos profissionais } \\
\text { de nível superior da Rede de Atenção Primária } \\
\text { à Saúde de Aracaju - SE. }\end{array}$ \\
\hline
\end{tabular}

REAS/EJCH | Vol.Sup.34 | e1413 | DOI: https://doi.org/10.25248/reas.e1413.2019 Página 6 de 9 
A Síndrome de Burnout (SB) acomete principalmente as mulheres, como foi observado no estudo de Kolhs $M$, et al. (2016) que mostra que houve um predomínio da SB no sexo feminino (89\%). No cuidado de enfermagem, a maioria dos profissionais são mulheres, esta associação ocorre desde sempre, o feminismo e o cuidado. Em relação ao estado civil dos enfermeiros pesquisados $55,5 \%$ apontaram serem casados e $44,5 \%$ solteiros.

A faixa etária predominante era entre 20 aos 39 anos (89\%) e 11\% com mais de 40 anos. Em estudo realizado foi verificado que a maioria dos profissionais possuía faixa etária abaixo de 41 anos, e relacionam que isto se deve ao fato da profissão de enfermagem estar crescendo nas últimas décadas, aumentando a procura pela profissão de pessoal mais jovem (WORM FA, et al., 2016).

Dessa forma, o estado civil do individuo também influência, pois existe aquela pressão interna, os filhos, o relacionamento em si, a síndrome afeta principalmente enfermeiros jovens, visto que por serem jovens sofrem mais cobranças a respeito do cumprimento de metas, muitos estão no meio da sua qualificação e acabam amontoando responsabilidades além do trabalho, o que acaba contribuindo para o esgotamento.

Estudos apontam que os profissionais mais jovens acabam por desenvolver mais frequentemente esta síndrome, por que além de estarem recém-formados, existe também o medo do novo, a inexperiência. O que está de acordo com o estudo de Tavares KFA, et al. (2014), que apontam que a média de idade mais afetada pela SB é 28 anos, em sua maioria enfermeiros recém-formados, e que estão se inserindo no mercado de trabalho.

Neste cenário, o enfermeiro recém graduado, ainda inseguro da prática e pressionado por resultados, torna-se mais susceptível a SB, visto que a inexperiência profissional, aliado a pouca idade, acaba influenciando na saúde desses enfermeiros, devido ao estresse causado pela inserção no mercado de trabalho (TAVARES KFA, et al., 2014).

De acordo com Kolhs M, et al. (2016) os profissionais de enfermagem de uma ou outra forma desenvolvem o desgaste emocional, quando no desenvolvimento das atividades nos setores que assiste aos pacientes oncológicos. Apresentando alguns sentimentos, como por exemplo: ansiedade, medos, inseguranças, incertezas, impotência e o peso do cuidado, sendo que a relação entre paciente e enfermeiro possibilita a ajuda no convívio. Todavia, esta convivência em muitos momentos demanda não somente o conhecimento técnico-científico, pois são momentos que se apresentam carregados de anseios, possibilitando o desgaste emocional além do físico nestes profissionais.

Profissionais que cuidam de pacientes em tratamentos paliativos, também são os que mais são afetados, pois lidam diariamente com o estresse ocasionado pelo tratamento deste tipo de paciente, visto que a incompreensão por parte dos familiares acaba por pressionar os enfermeiros por melhoras imediatas e definitivas, o que é algo que não está nas mãos destes profissionais. Há também o desgaste emocional do enfermeiro, por causa do envolvimento com o paciente em tratamento, onde muitas vezes as emoções do profissional se misturam com a emoção e dor do paciente (TAVARES KFA, et al., 2014).

Neste mesmo contexto, em um estudo para mapear os fatores de risco de desenvolvimento da Síndrome de Burnout nos profissionais de Enfermagem da Equipe do Serviço de Atendimento Móvel de Urgência, realizado por Worm FA, et al. (2016), dentre os 18 participantes $78 \%(n=14)$ eram técnicos de enfermagem e $22 \%(n=4)$ enfermeiros, com predomínio do gênero feminino $(77,78 \%)$, faixa etária entre 25 e 31 anos (33,33\%). A metade dos participantes (50\%) era casada e a casado e maioria possui filhos (WORM FA, et al., 2016).

Vale mencionar que os profissionais que trabalham na urgência estão mais susceptíveis ao desenvolvimento desta síndrome, visto que além do estresse emocional, há também o desgaste físico, pois estes profissionais trabalham muito com o corpo, onde ele trabalha muitas vezes em locais de risco, sem segurança, o pensamento e tomada de decisão de forma rápida, acaba por influenciar no desenvolvimento desta síndrome (WORM FA, et al., 2016). No entanto, os enfermeiros são os profissionais que ficam na frente de trabalho, são eles que mais lidam diretamente com os pacientes, isso aliado à longas horas de trabalho, 
plantões intermináveis e a cobrança por parte dos superiores, acarreta um estado de estresse permanente que vai aumentando até a exaustão psicológica, física e emocional. Os conflitos dentro da equipe também são considerados um fator predisponente para o desenvolvimento da síndrome de Burnout (SILVA SCPS, et al., 2015).

O que corrobora com o estudo de Campos ICS, et al. (2015) que apontou que os enfermeiros e técnicos de enfermagem constituem uma equipe com grande chance de predisposição ao desenvolvimento desta síndrome, por serem os profissionais de saúde que mais tempo ficam em contato com o paciente e seus familiares. Além disto, no âmbito hospitalar, esses profissionais são submetidos a condições insalubres e precárias de trabalho, que geram uma baixa qualidade de vida.

Na concepção de Pereira MMA e Gomes ARS (2016) dentre os fatores que podem contribuir para a SB, estão o estresse ao se lidar com os clientes/pacientes, a busca pela melhoria na carreira, problemas com relação à remuneração e a sobrecarga de trabalho, relacionado à SB, existe o esgotamento emocional e a baixa satisfação pessoal.

A depressão é um fator de risco para o desenvolvimento da Síndrome de Burnout, e acaba por camuflar um distúrbio ainda mais grave, muitos profissionais negam até para si mesmo, a presença desta doença, mesmo sendo um conhecedor desta condição. Em estudo realizado para medir os índices de depressão, mostrou- se que $84,4 \%$ dos profissionais não admitiram problemas de depressão e ansiedade, $12,8 \%$ disseram apresentar depressão leve e 10,7\% disforia. Em relação dos estados depressivos, 3,5\% mostraram índices de depressão moderada, não foram encontrados casos de depressão grave (SEGUEL F, et al., 2016).

Para Silva JLL, et al. (2015) salienta que o esgotamento emocional, é outro fator relevante no que diz respeito à qualidade de vida dos enfermeiros. Pois, os níveis elevados de esgotamento emocional, fator central do esgotamento profissional, levam a uma queda da qualidade de saúde e de vida, ao desgaste emocional e à sensação de falta de energia, mostrando associação inversa com desempenho no trabalho.

Quando o profissional apresenta esta síndrome e não busca tratamento, o rendimento laboral cai a nível alarmante, não correspondendo ao nível de conhecimento pertencente a ele, desta forma a síndrome interfere negativamente na carreira profissional, bem como no rendimento pessoal (SEGUEL F, et al., 2016).

Uma melhor atenção deve ser dada para as manifestações da Síndrome de Burnout (SB) nos profissionais enfermeiros do serviço público, onde muitas vezes se impõe exigências, tarefas e habilidades específicas com a população. Na Atenção Primária, os profissionais além da enorme demanda do trabalho, também lidam diariamente com a doença e o sofrimento subjetivo e sintomas somáticos (SILVA JLL, et al., 2015).

Mesmo profissionais que trabalham em ambientes considerados mais tranquilos em relação a cobranças como, por exemplo, enfermeiros da atenção primária, podem desenvolver esta síndrome, porque mesmo sem a constante cobrança dos superiores, estes profissionais recebem a cobrança direta dos clientes/ pacientes com relação à resolutividade, lidam também com um grande número de pacientes por dia (LIMA AS, 2016).

Segundo Silva CCL, et al. (2016), o acúmulo de responsabilidades, carga de trabalho e deveres de complexidades diferentes exigem demandas físicas e psicológicas que vão além do suportado pelo trabalhador. Destacam-se, desta forma, aspectos do trabalho que podem contribuir para o desenvolvimento da SB: pressão por metas, fragmentação de tarefas, questões administrativas, ambientais e de relacionamento, competitividade, baixa autonomia, insegurança no trabalho, sobrecarga, alta demanda, falta de trabalho em equipe e a repetitividade de tarefas.

Desse modo, o trabalho dos enfermeiros na ESF frente as precárias condições sociais do território, a desarticulação da rede de saúde e a cobrança profissional pelos gestores ou pelo próprio trabalhador acaba auxiliando no desenvolvimento desta síndrome.

Trabalhar com que gosta, representa um fator importante na escolha da profissão, pois a satisfação está diretamente liga da com o bem rendimento e realização do trabalho, quando esta satisfação não existe, temse profissionais irritadiços, desmotivados e principalmente exaustos emocionalmente (LIMA AS, 2016). 
Conforme Sá MAS, et al. (2014), ressaltam que quanto menor a satisfação, maior é a probabilidade de desenvolvimento de Exaustão Emocional e de Despersonalização e menor é a possibilidade do indivíduo se sentir realizado profissionalmente. No contexto atual, o trabalho é considerado como um espaço social de grande importância para vida do indivíduo, uma vez que contribui em grande parte com a formação da identidade do indivíduo, assim como com as maneiras de interação entre estas pessoas.

\section{CONSIDERAÇÕES FINAIS}

A partir do estudo foi possível compreender, explorando a bibliografia já existente, os fatores que podem influenciar no desenvolvimento da síndrome de Burnout (SB), os principais sintomas apresentados e devido à mesma impactar e interferir na qualidade de vida dos profissionais. Atualmente, as pessoas investem uma parcela de sua vida na preparação e dedicação na sua atividade laboral. Portanto, o trabalho, além de possibilitar o crescimento pessoal, as transformações, o reconhecimento, a independência pessoal e profissional, o mesmo poderá causar sérios problemas, os quais podem afetar diretamente nasua saúde mental como também pode prejudicar a qualidade do desempenho de sua função empregatícia proporcionada por está patologia se não identificada, diagnóstica e tratada precocemente.

\section{REFERÊNCIAS}

1. CARRARA GLR, et al. Assistência de enfermagem humanizada em saúde mental: uma revisão da literatura. Rev Fafibe On-Line, São Paulo, 2015; 8(1): 86-107.

2. CAMPOS ICS, et al. Fatores Sociodemográficos e Ocupacionais Associados à Síndrome de Burnout em Profissionais de Enfermagem. Psychology/Psic Ref Crít, Minas Gerais, 2015; 28(4): 764-771.

3. FRANÇA TLB, et al. Síndrome de Burnout: características, diagnóstico, fatores de risco e prevenção. Rev enferm UFPE on line, Recife, 2014; 8(10): 3539-3546.

4. KOLHS M, et al. Sentimentos de Enfermeiros Frente ao Paciente Oncológico. J Health Sci, Rio Grande do Sul, 2016 ; 18(4): 245-250.

5. LIMA AS. Prevalência e fatores associados à síndrome de Burnout nos profissionais da saúde da atenção primária de juiz de fora, 2016, 139 f. Dissertação (Mestrado em Saúde Coletiva) - Faculdade de Medicina da Universidade Federal de Juiz de Fora. Juiz de Fora, 2016.

6. MENDES KDS, SILVEIRA RCCP, GALVÃO CM. Método de pesquisa para incorporação de evidências na saúde e na enfermagem: Revisão integrativa. Texto contexto-enfermagem, Florianópolis, 2008; 17(): 758-764.

7. PEREIRA MMA, GOMES ARS. Stress, Burnout e avaliação cognitiva: estudo na classe de Enfermagem. Arq Bras de Psic, Rio de Janeiro, 2016; 8(1): 72-83.

8. RODRIGUES UMP, RIBEIRO ER. Síndrome de Burnout na equipe de saúde da família: revisão de literatura. Rev Saúde e Desenvol, São Paulo, 2014; 5(3): 168-181.

9. SANTOS AVS, et al. Sentimentos e dificuldades do familiar do idoso com transtorno mental, Atas CIAIQ, São Paulo, 2016; 2(1): 1060-1069.

10. SEGUEL F, et al. A Síndrome de Burnout em trabalhadores de enfermagem em dois hospitais no sul do Chile. Av Enferm, Chile, 2016; 34(1): 39-47.

11. SILVA CCS, et al. Burnout e tecnologias em saúde no contexto da enfermagem na Atenção Primária à Saúde. Esc Anna Nery, Rio Grande do Norte, 2016;21(2): 1-7.

12. SÁ MAS, SILVA PO, FUNCHAL B. Burnout: o impacto da satisfação no trabalho em profissionais de enfermagem. Psicologia \& Sociedade, Vitória-ES, 2014; 26(3): 664-674.

13. SILVA JLL, et al. Fatores psicossociais e prevalência da síndrome de Burnout entre trabalhadores de enfermagem intensivistas. Rev Bras Ter Intensiva, Rio de Janeiro, 2015; 27(2): 125-133.

14. TAVARES KFA, et al. Ocorrência da síndrome de Burnout em enfermeiros residentes. Acta Paul Enferm, São Paulo, $2014 ; 27(3): 260-265$.

15. WORM FA, et al. Risco de adoecimento dos profissionais de enfermagem no Trabalho em atendimento móvel de urgência. Rev Cuid, Santa Catarina, 2016; 7(2): 1288-96. 